\title{
Performance of wide-area power system stabilizers during major system upsets: investigation and proposal of solutions
}

\author{
Mokhtar Benasla $^{1^{*}}$, Mouloud Denaï ${ }^{2}$, Jun Liang ${ }^{3}$, Tayeb Allaoui ${ }^{1}$, Mostefa Brahami ${ }^{4}$, \\ ${ }^{1}$ L2GEGI Laboratory, Electrical Engineering Department, Ibn Khaldoun University, Tiaret, Algeria \\ ${ }^{2}$ School of Physics, Engineering and Computer Science, Hertfordshire University, Hatfield, UK \\ ${ }^{3}$ School of Engineering, Cardiff University, UK \\ ${ }^{4}$ ICEPS Laboratory, Department of Electrotechnics, Djillali Liabes University, Sidi Bel Abbes, Algeria \\ ${ }^{*}$ Corresponding author. Email: benasla.mokhtar@yahoo.fr
}

\begin{abstract}
Wide-area damping controllers (WADCs) are effective means of improving the damping of inter-area oscillations and thereby ensuring a secure operation of modern highly stressed interconnected power systems; however, their implementation costs are high. Therefore, the controller must be well configured and designed to ensure its cost-effectiveness. Several techniques have been proposed in the literature to design effective controllers and good results have been achieved. However, some important practical aspects that could potentially impact the performance of the designed controller have not been addressed or studied in sufficient detail in these previous works. One such aspect is assessing the performance of the designed controllers under major system upsets resulting in large deviations in the frequency and fluctuations in the power. These may lead to controller saturation which could negatively impact its damping performance or even cause instability. In this paper, the impact of such large upsets is investigated on several test systems via extensive small- and large-signal analyses and it is shown that, during severe transients, controller saturation may occur and persist over a long period of time, posing a potential threat to the power system stability. This paper presents a very effective solution to alleviate this problem and help design more robust WADCs. The simulation results show that the proposed solution works well and leads to improved power system stabilisers performance during transient upsets.
\end{abstract}

Keywords: Power system stabilizer; inter-area oscillations; wide-area damping controller; saturation limits; transient stability

\section{Introduction}

With the ever-increasing complexity of interconnected electric power systems networks, several different forms of stability issues have emerged and became problematic for grid operators. However, transient stability remains a major area of concern in the design of stable, reliable and secure operation of power systems [1], [2]. Traditionally, Power System Stabilizers (PSS) have been used to inject auxiliary stabilizing signals derived from rotor speed, power, or terminal frequency to provide maximum damping of power system oscillations [3, 4]. However, experience has shown that PSSs do not always ensure that the potential of the excitation system (AVR: Automatic Voltage Regulator) for improving transient stability is fully exploited [5]-[7]. The main reason for this is that, under certain conditions, the PSS can reduce transient stability by overriding the voltage signal to the exciter. One of the most critical cases occurs when the power system is subjected to large disturbances (e.g. load rejection or loss of generation) causing large frequency offsets. In this case, the PSS leads to excessive terminal voltage and reactive power deviations which adversely affects the system transient performance. In some systems, following a severe fault, the PSS output is immediately disconnected to prevent it from competing with the action of the voltage regulator [6], [8]. During severe events, the PSS output can be driven into saturation and remains in this state for a while. Over this period, the PSS becomes ineffective which put the power system stability under a potential threat. Furthermore, during these saturation intervals, the PSS deteriorates the dynamic performance of the excitation system [8], [9].

With the increasing size and complexity of the interconnected systems, inter-area oscillations have become a major concern. The stabilization of these electromechanical oscillations between interconnected synchronous generators (inter-area modes) is vital for a secure operation of the system. PSSs are commonly employed for enhancing the damping of inter-area modes [2]. Inter-area oscillations often involve many sub-networks and consequently, to implement the most effective solution, the cooperation/contribution of all these sub-networks will be required. In recent years, there has been an increasing trend towards the use of wide-area controllers for damping inter-area oscillations in power systems. It was found that if remote signals are applied to PSS controller design a better damping of the inter-area oscillations can be achieved thus enhancing the system dynamic performance [10]. Owing to the recent advances in fibre-optic telecommunications and global positioning systems, many electric power utilities in North America and Europe have started to adopt wide area monitoring and control technology [11], [12]. Based on wide area signals, different methods are presented in the literature to design effective PSSs [12]-[20]. 
However, the inherent delays associated with wide-area feedback signals negatively impact the performance of the designed controllers. These aspects must to be carefully considered in the control design. Most published studies on wide-area PSS, sometimes referred to as Wide-Area Damping Controller (WADC) or Global PSS, deal mainly with the improvement of system damping without a thorough assessment of its performance during major system upsets. The effects of communication delays have been extensively investigated in the literature, and various methods have been proposed considering time delay in the design of WADCs [21-25]. However, the effects of saturation have been addressed only by a few authors [26-29]. As discussed in [3], [30], one of the specific objectives of excitation control design is to prevent adverse effects on system performance during major system upsets that cause large frequency and voltage excursions. For PSSs based on local input signals, the excursion problem is well-recognized and well-documented and the problem can be alleviated by using a dual-input stabilizer (PSS2A and PSS2B in IEEE Std 421.5 [5], [31]) which combines electrical power and speed deviations as input signals to derive the stabilizing signal. For PSSs using remote input signals, the excursion problem has not been addressed on an ad hoc basis [27-29]. This paper seeks to investigate this problem with detailed analysis. The performance of wide-area PSSs during major system upsets is investigated using different well-known power system benchmarks, and some important solutions are proposed to effectively overcome the associated problems.

The paper is organized as follows. Section 2 briefly provides some background on the linear analysis of power systems. The problem is formulated in Section 3. The proposed potential solution to the problem is presented in Section 4 . Section 5 describes the application of the proposed solution. Section 6 presents some discussions, and Section 7 summarises the conclusions of the paper.

\section{Background}

A power system is a nonlinear system which can be usually linearised around a given operating point. This linear behaviour is very useful for the analysis of system oscillations. In addition, linear models can be used to design controls that damp system oscillations [32], [33]. The linearised model of the power system can be described by the following state equation:

$$
\begin{aligned}
& \dot{x}=A x+B u \\
& y=C x
\end{aligned}
$$

where $x, u$ and $y$ are the state, input and output vectors, respectively. $A, B$ and $C$ are state, input and output matrices, respectively. An eigen analysis of matrix A produces the eigenvalues $\lambda_{i}$ and their corresponding right and left eigenvectors $e_{i}, v_{i}$, respectively. Physically, the right eigenvector gives the relative activity of the state variables when a particular mode is excited. It is known as the mode shape in the literature. The mode shape provides important information on the participation of an individual machine or a group of machines in one particular mode.

The controllability of mode $i$ from the $j^{\text {th }}$ input is given by:

$$
K_{c}=\left|v_{i} B_{j}\right|
$$

The observability of mode $i$ from the $j^{\text {th }}$ output is given by:

$$
K_{o}=\left|C_{j} e_{i}\right|
$$

System (1) can be expressed by:

$$
\frac{y(s)}{u(s)}=C(s I-A)^{-1} B=\sum_{i=1}^{n} \frac{R_{i}}{s-\lambda_{i}}
$$

where $R i$ is the residue associated with mode $\lambda_{i}$ and represents an index containing the controllability and the observability information. It is given by [13]:

$$
R_{i}=C e_{i} v_{i}^{T} B
$$

Since the residue gives the sensitivity of the corresponding mode to the feedback of the transfer function output to its input, the PSS is located at the generator having the largest residue magnitude [34]. Therefore, the signal with highest observability is chosen as input to the PSS. The angle of the residue indicates the required phase compensation for each mode to achieve damping effect.

The required phase compensation for mode $\lambda_{i}$ is given by [13]: 
As mentioned above, the power system is a nonlinear system. Therefore, the performance of the controller (PSS) which is designed using linear model can be limited due to the inherent power system nonlinearities [26-29].

\section{Problem formulation}

The AVR is primarily used for regulating the terminal voltage of the generator to which it is attached by changing the generator field voltage. The PSS is then introduced to provide the required damping by modulation of the generator field voltage. Since both strategies (voltage regulation and damping improvement) are performed via the field voltage, it is impossible to achieve both goals simultaneously [5, 35]. Let us illustrate the adverse effect of PSS on the performance of an AVR during large frequency excursions using the well-known test system shown in Fig. 1. Details on the system model and its parameters values can be found in [4]. Each generator is equipped with a static exciter (high gain: 200) and a steam turbine governor. The load is represented as constant impedances and the nominal system is operating with Area 1 exporting 413 MW to Area 2. The model is linearised around a nominal operating point. Small signal analysis shows that the system has three electromechanical modes (see Table 1). In order to damp these oscillations, PSSs are required.

\subsection{PSS based on local signal}

An original PSS is attached to generators G2 and G4. The block diagram of the PSS is shown in Fig. 2. It was designed in [4], [36] to enhance the damping of the local and inter-area modes of the system under consideration. Table 2 clearly shows that the addition of the stabilizers improves the damping of all three modes. However, the PSS adversely affects the terminal voltage during severe disturbances. Fig. 3(a) shows the terminal voltage of G1 and G2 ( $V_{1}$ and $\left.V_{2}\right)$ when the load at bus 7 is increased at time $1 \mathrm{~s}$ by $10 \%$. It is clear from this figure that the terminal voltage of G1 (AVR without PSS) is effectively controlled and the AVR demonstrates a good voltage recovery capability, while the AVR at G2 (with PSS) showed some weaknesses and the voltage recovery is not good due to the supplementary damping signal ( $\mathrm{V}_{\mathrm{S} 2}$ ) provided by the PSS (see Fig. 3(a)).

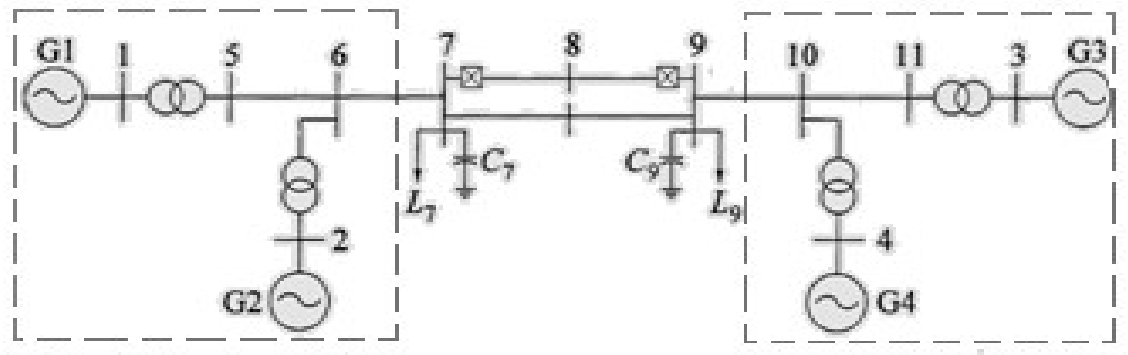

Area 1

Area 2

Fig. 1 Two-area system.

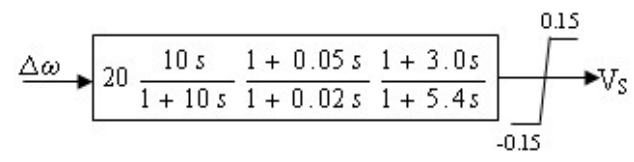

Fig. 2 PSS block diagram.

Table 1. Electromechanical modes of oscillation.

\begin{tabular}{llccl}
\hline Mode & Eigenvalue & $\begin{array}{c}\text { Freq. } \\
(\mathbf{H z})\end{array}$ & Damping & Mode shape \\
\hline Locale mode (Area 1) & $-0.681 \pm \mathrm{j} 7.021$ & 1.11 & 0.09 & G1 vs G2 \\
\hline Local Mode (Area 2) & $-0.676 \pm \mathrm{j} 7.243$ & 1.15 & 0.09 & G3 vs G4 \\
\hline Inter-Area Mode & $0.104 \pm \mathrm{j} 4.022$ & 0.64 & -0.02 & (G1,G2) vs (G3,G4) \\
\hline
\end{tabular}

Table 2. Effect of PSS at G2 and G4.

\begin{tabular}{ccc}
\hline & Freq & Damp \\
\hline Local Mode (Area 1) & 1.21 & 0.21 \\
\hline Local Mode (Area 2) & 1.25 & 0.23 \\
\hline Inter-Area Mode & 0.65 & 0.04 \\
\hline
\end{tabular}


During system disturbances involving excessive frequency excursions (e.g. large loss-of-generation event), the PSS output can go into saturation for a while, which causes the unit to respond to the system event in a manner detrimental to stability [9]. This is illustrated in Fig. 3(b), which shows the results when $50 \%$ of load at bus 9 is tripped. It has been found that this problem can also occur if electrical power is used as a stabilizing input signal $[8,37]$.
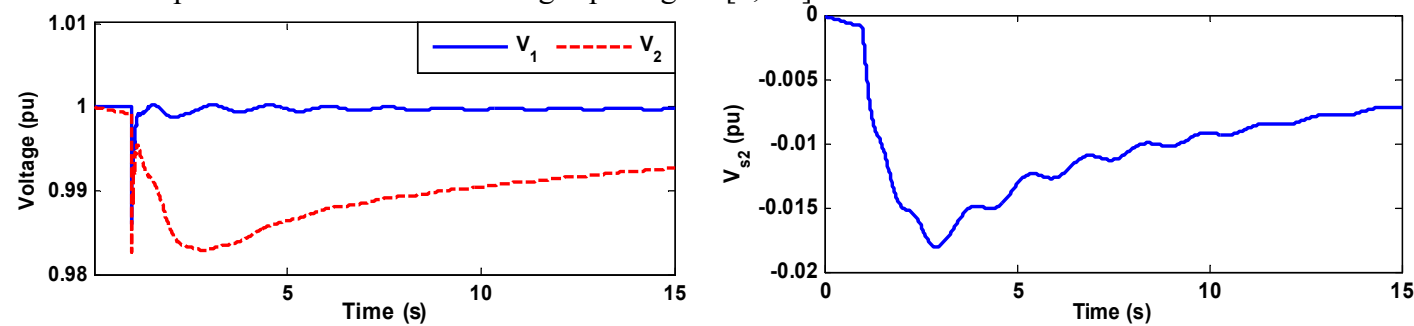

(a)
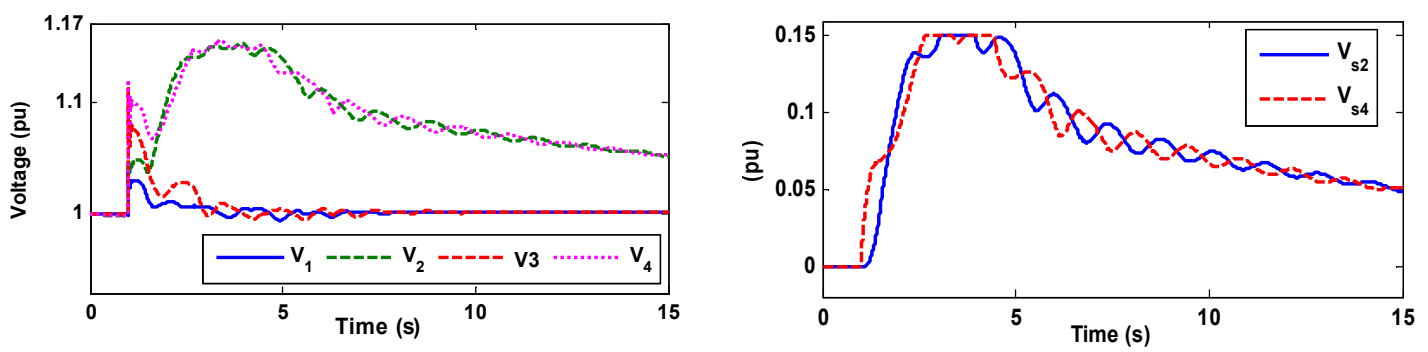

(b)

Fig. 3 Response of the PSS during transient conditions, (a) Adverse impact of PSS on the terminal voltage (b) Saturation of the PSS following a large frequency offset.

It should be emphasized that some techniques have been proposed to mitigate the adverse impact of the PSS on voltage control when there is a large frequency offset. However, all these solutions are restrictive [37]. For example, if the stabilizer output is immediately disconnected from the regulator following a severe fault, this does not degrade the action of the AVR, but will disable the PSS for a long period of time, during a post-contingency phase where it is most needed, because a well-damped response following severe system upset is desirable [3,7]. For example, on many occasions on the Ontario Hydro system has experienced immediate loss of stability of the generator following the disconnection of the stabilizing signal [38].

\subsection{PSS based on remote signals}

It is well known that conventional PSSs may not be effective in damping inter-area modes under certain situations. The main reason is-because inter-area modes are not as highly controllable and observable in the generator's local signals as the local modes. The inter-area modes are either weakly observable or weakly controllable by local stations. For this reason, the widearea PSSs are proposed to improve the damping of the critical inter-area modes. Observability and controllability concepts are commonly used to select the optimal signal to be measured and the appropriate location for the controller. Since the rotor speed, electrical power and terminal frequency (taken from remote measurements) are among the commonly used input signals, the wide-area PSS will also adversely affect the terminal voltage during severe system upsets and the saturation problem will arise, especially when the measurement location is close to the disturbance point. Then the wide-area PSS can be responsible for the propagation of the disturbance from one area to another in a large interconnected power system. In order to investigate this problem, consider the system shown in Fig. 1. In this system the inter-area mode is strongly observable in area 2 (G4 and G3), and strongly controllable in G2 (the system input and output are the exciter inputs and rotor speeds, respectively), and consequently it can be seen form Table 2 that the PSSs (at G2 and G4) do not provide substantial damping improvement for the inter-area mode, because this mode is not well observable in G2. The corresponding frequency responses in Fig. 4(a) clearly confirm that G2 has a little impact on the inter-area mode. One solution for this problem is to improve the observability by using a remote signal from Area 2 as input to the PSS at G2 (as in [10]). This is confirmed by Fig. 4(b). It was found that the observability vectors from the rotor speeds in Area 2 have larger magnitudes than those through rotor speeds in Area 1, but with a phase around $180^{\circ}$ (i.e. the generators in one area are almost in anti-phase with those in the other area). Then, the speed of a generator in Area 2 can be used as an input to a PSS in Area 1 by using a negative gain [34]. It can be seen from Table 3 that the damping of the inter-area mode is significantly improved when a remote signal ( $\Delta \omega$ of G3) is used as input to the PSS at G2; however, the damping of the local mode of Area 1 has been reduced because this mode is not observable in Area 2 (see Fig. 4(b)). Fig. 5(a) demonstrates the improved damping achieved by the remote signal (Remote) when $20 \%$ of load at bus 9 is tripped; however, due to the large magnitude of the observability index of the inter-area mode, the adverse effect on terminal voltage is greater than that of the local signal (Local). As can be seen from Fig. 5(b), the PSS saturates for several seconds when $35 \%$ of load is tripped. During this period the PSS is inactive, and the generator oscillates over a longer period of time. 


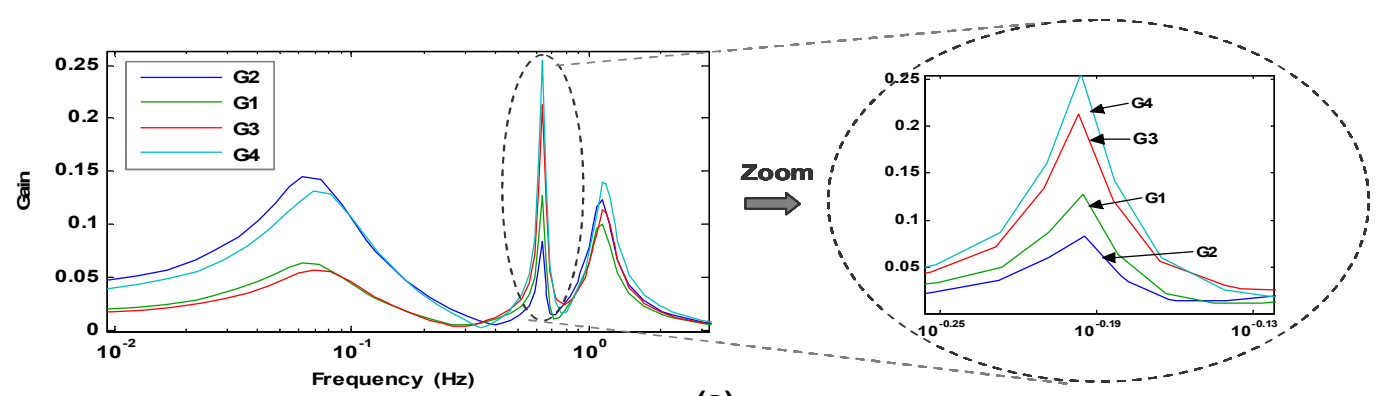

(a)

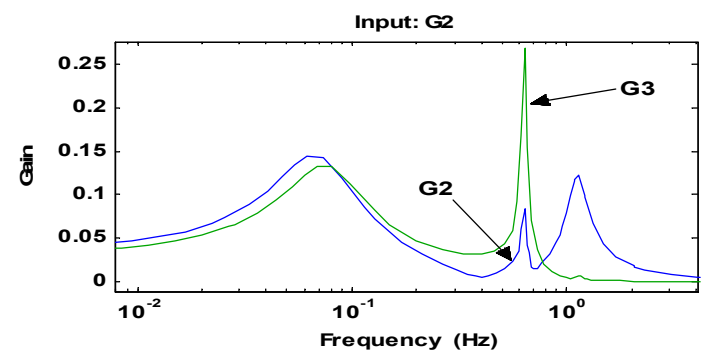

(b)

Fig. 4 Frequency responses. (a) single-input single-output frequency response. (b) single-input multi-output frequency response (Input: G2).

\begin{tabular}{ccc}
\hline & Freq & Damp \\
\hline Local Mode (Area 1) & 1.11 & 0.11 \\
\hline Local Mode (Area 2) & 1.22 & 0.22 \\
\hline Inter-Area Mode & 0.65 & 0.12 \\
\hline
\end{tabular}

Table 3. Effect of remote signal on damping.
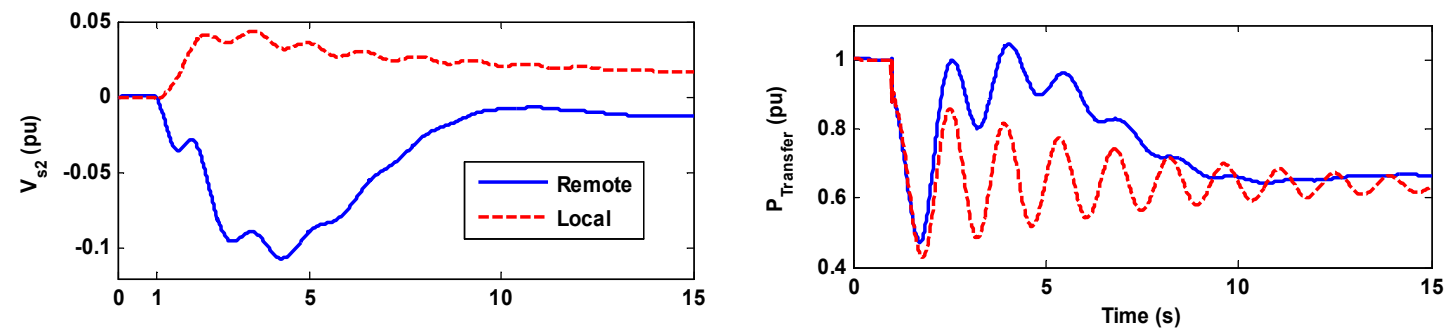

(a)
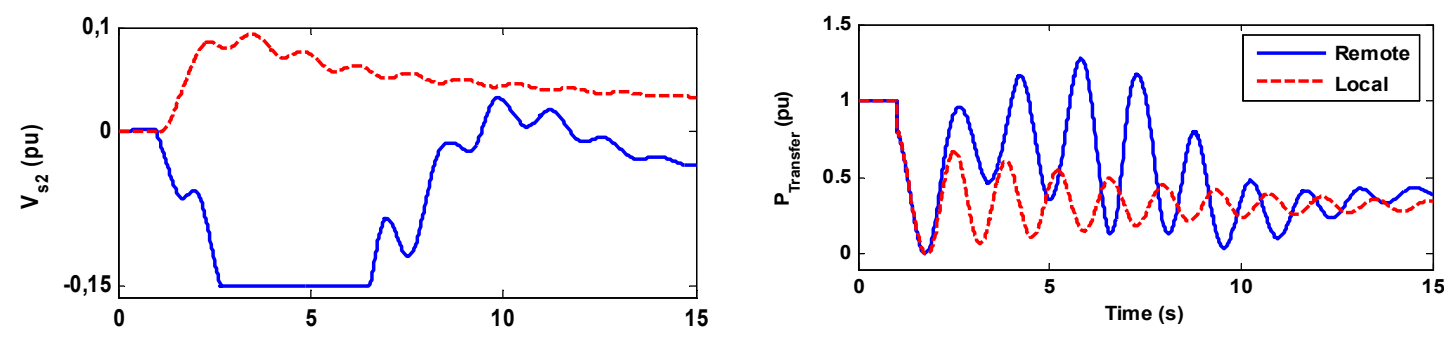

(b)

Fig. 5 Response of the PSS with a remote signal. (a) Adverse impact of PSS with remote signal (P $\mathrm{P}_{\text {Transfer: }}$ active power transfer from area 1 to area 2). (b) Saturation of the PSS with remote signal.

The large magnitude of the observability index of the inter-area mode when a remote signal is used implies that small stabilizer gain can provide satisfactory performance while minimizing the adverse impact of the PSS on voltage control and consequently reducing the likelihood of PSS saturation as can be seen in Fig. 6. However, the stabilizer gain should be set such that to produce maximum damping of the critical mode [3,30], especially for wide-area PSSs to justify the communication 
infrastructure costs. Table 4 shows the effect of increasing the stabilizer gain on the inter-area mode and the two local modes. It is seen from the results that increasing the stabilizer gain improves the damping of the inter-area mode.

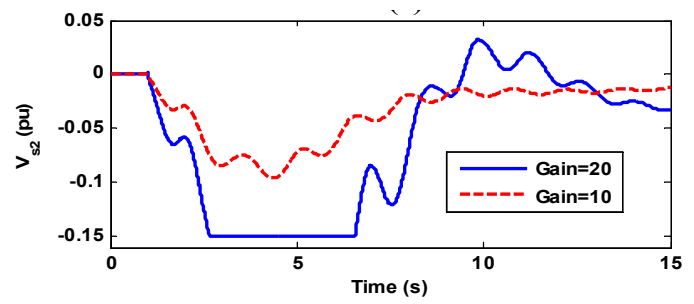

Fig. 6 Effect of changing stabilizer gain.

\begin{tabular}{cccc}
\hline PSS gain & Local mode 1 & Local mode 2 & $\begin{array}{c}\text { Inter-area } \\
\text { mode }\end{array}$ \\
\hline 5 & 0.09 & 0.23 & 0.04 \\
\hline 10 & 0.09 & 0.22 & 0.07 \\
\hline 15 & 0.11 & 0.22 & 0.09 \\
\hline 20 & 0.11 & 0.22 & 0.12 \\
\hline
\end{tabular}

Table 4. Effect of increasing PSS gain on damping.

It can be seen that the same damping (as with local signal, see Table 2) of the inter-area mode can be achieved with a gain of only 5. This demonstrates the significant benefit in control design when more information is available. It should be noted that additional performance can be obtained by an appropriate retuning of the PSS. However, this will not solve the excursion problem because this problem usually is not taken into account when designing or tuning PSSs. Generally, PSS tuning must be done to obtain maximum damping, and then the offset problem is limited by applying the restriction techniques (employ low PSS gain or output limits, disconnect the PSS with a small washout time constant) where the effectiveness of the PSS in damping system oscillations is severely limited by these techniques. The wide-area PSS is an expensive solution for damping specific inter-area modes, and therefore it is unacceptable to restrict its effectiveness by any techniques. Moreover, the widearea PSS is used to improve the damping of the critical inter-area modes (e.g. the situation where the power transfer level is limited by inadequate damping of power oscillations after certain N-1 post-contingency conditions) and if it is deactivated following a severe disturbance due to a severe saturation or a programmed washout effect, it would be a serious threat to the safe and stable operation of the system. Recently, some techniques have been proposed to handle the saturation problem in WADCs [27-29]. However, these proposed techniques do not address the core of the problem, since they are based on the concept of "minimum energy control". They overcome the saturation problem but do not ensure that the controller provides its maximum possible damping, which is highly desirable during transient periods. Hence, it is necessary to find an acceptable solution to this problem in order to ensure cost-effectiveness of the wide-area PSSs. To achieve this objective, the only possible solution is to find a good input signal which can maintain a good control performance during major system upsets. This is discussed in the next section.

\section{Possible solution}

Since the main purpose of wide-area PSS is to improve the damping of a critical inter-area mode, the selected signal should provide sufficient observability of this mode. Due to the response of the power system to extreme contingencies and major upsets, it may be difficult or even impossible to find the desired signal which should have good observability to the interarea mode of concern while achieving a good PSS performance during major system upsets. Experience with alternative stabilizing signals has shown that the offset problem produced by the PSS is greatly reduced when the accelerating power is used as input signal [37-39]. Accelerating power signal is the result of a combination of electrical and mechanical power signals as shown in equation (7).

$P_{a}=P_{m}-P_{e}$

where $P_{m}$ is the mechanical power, $P_{e}$ is the electrical power and $P_{a}$ is the accelerating power. The electrical power signal is used to provide the necessary damping while the mechanical power signal is used to reduce the terminal voltage and Var offsets during large disturbances. Based on the same principle, the desired signal may be derived by combining two signals.

After a system upset, the frequencies of the different generators operating in synchronism in a large interconnected system will vary in the same direction (see Fig. 7), and generally they can be shown as relatively small variations over an average frequency in the system [40-42]. Therefore, if the speed difference signal $\left(\Delta \omega_{j}-\Delta \omega_{k}\right)$ of machines $j$ and $k$ is used as input, one can expect 
that the excursion problem will be solved. Fig. 8(a) shows the good response of the combined signal in comparison with the individual signals when the load at bus 7 is increased by $5 \%$. Therefore, the PSS will not affect the terminal voltage if the combined signal is used as input and the saturation problem will be avoided. The PSS with the combined signal will give an excellent performance during major system upsets in contrast to the PSS2A which required a ramp-tracking filter to track rapid rates-of-change in the measured electrical power signal, because mechanical power changes are slower in practice, even for fast valve movements.

As a second requirement of the desired signal, the combined signal should provide high observability for the inter-area mode of interest. If the observability indices of the mode of interest from the rotor speeds of the two machines $j$ and $k$ have an opposite sign, then the magnitude of the observability index from these two machines speed difference signal will be larger than each of the individual observability indices (of course, the opposite is true).

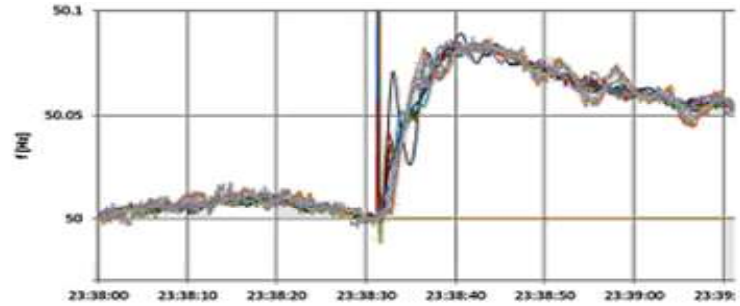

(a)

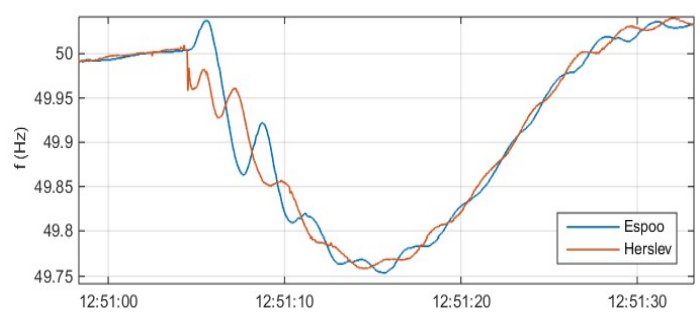

(b)

Fig. 7 Recording of system frequency after severe upsets in the ENTSO-E system (Source: ENTSO-E). (a) Frequency at different locations after loss of load (2000 MW). (b) Frequency at two locations (Espoo: Southern Finland and Herslev: Denmark) after a loss of 580 MW.

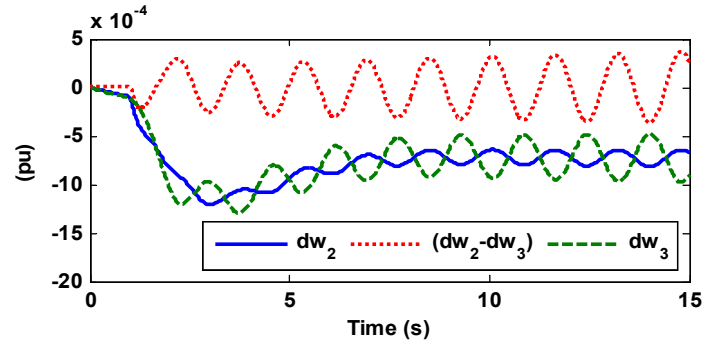

(a)

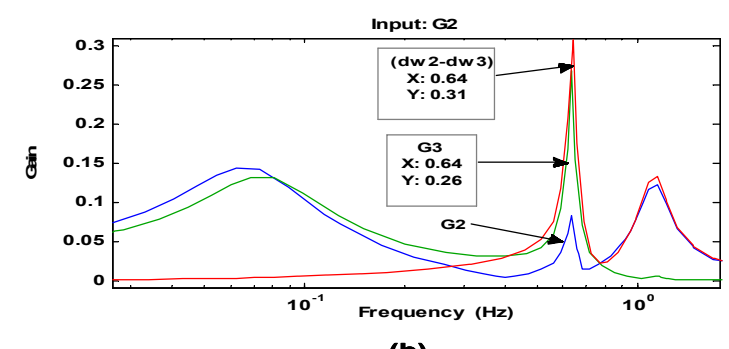

(b)

Fig. 8 Benefits of the combined signal. (a) Responses of the combined signal and the individual signals. (b) single-input multi-output frequency response (Input: G2).

It is well known that inter-area oscillations appear as a group of generators in one area swinging against a group of generators in another area. Even in the large multi-area power systems, every mode (inter-area) still gives priority to two groups oscillating against each other [4]. The eigenvectors are used to determine the mode shape and to provide information about the network elements and generators which participate in each mode. Mode shapes are a very useful tool to identify the oscillation patterns of each inter-area mode. Equation (3) gives the observability index of mode $i$ form the $j^{\text {th }}$ output. The angle of the vector $C_{j} e_{i}$ (observability vector) gives the phase information of the oscillation and can be used to divide the generators into two groups which oscillate against each other for each inter-area mode. An inter-area mode may be strongly observable and strongly controllable in the same group or may be strongly observable in one group and strongly controllable in the other group (recall that the wide-area PSS is usually proposed for the last case). Generally speaking, if two machines in generator groups oscillating against each other in an inter-area mode (this is clearly shown in Fig. 7(b) and Fig. 8(a)), the observability vectors of this mode from the rotor speeds of the two machines are in opposite directions and the phase between the two vectors is always about $180^{\circ}$ [10], [13], [43]. Therefore, if a wide-area PSS is be used at machine $j$ (high controllability) using a remote signal from machine $k$ (high observability), the signal $\left(\Delta \omega_{j}-\Delta \omega_{k}\right)$ is the desired signal. The remote signal is used to provide the required damping of the critical inter-area mode, while the local signal is used to mitigate the PSS output deviation during severe upsets which enables the PSS to provide damping without adversely affecting the terminal voltage and reduces the probability of PSS saturation accordingly. Furthermore, the local signal will provide observability of the local modes and may also increase the observability of the inter-area mode of interest. The corresponding frequency responses in Fig. 8(b) clearly confirms that the combined signal is more effective to damp the inter-area mode than individual signals. Fig. 8(b) also shows that the combined signal has the desirable sensitivity to add damping to the local mode of Area 1 in contrast to the individual signal $\left(\Delta \omega_{3}\right)$, as can be seen in Table 5. It should be noted that alternative forms of wide-area PSS have been developed using combined signals as inputs $[12,13,43,44]$. However, in these works, the combined signals have been used because they provide a high observability of the inter-area modes; their advantage during system upsets is not investigated and is not known. 


\begin{tabular}{ccc}
\hline & Freq & Damp \\
\hline Local Mode (Area 1) & 1.16 & 0.27 \\
\hline Local Mode (Area 2) & 1.28 & 0.19 \\
\hline Inter-Area Mode & 0.66 & 0.12 \\
\hline
\end{tabular}

Table 5 Damping performance of combined signal.

\section{Application}

In order to investigate the performance of the proposed solution during transient upsets, we carried out the following test (it should be noted that the remote signal is always applied to the PSS at G2.):

A three-phase fault was applied in the middle of the tie-lines (bus 8 in Fig. 1) for $100 \mathrm{~ms}$ duration. The fault is cleared with no tie-line outage, but after $100 \mathrm{~ms}, 35 \%$ of load at Bus 9 is tripped. Fig. 9(a) shows the system response during this event. The disconnection of the load results in a considerable over-frequency, driving the PSS (with individual input signal) into saturation for several seconds causing the generator terminal voltage to decrease (due to the negative PSS gain, the voltage is depressed and not raised). Under these kinds of events, any stabilizer will tend to move the voltage in the wrong direction and reduce transient stability performance [7,9]. During severe events, the PSS may fail or operate improperly which can result in a unit trip [9], as can be seen in Fig. 9(b) where G1 is tripped. It is clear from this figure that the PSS fails to recover from saturation, thereby losing the dynamic control of the voltage and the system loses synchronism after $13 \mathrm{~s}$. It is then necessary to disconnect the stabilizing signal.
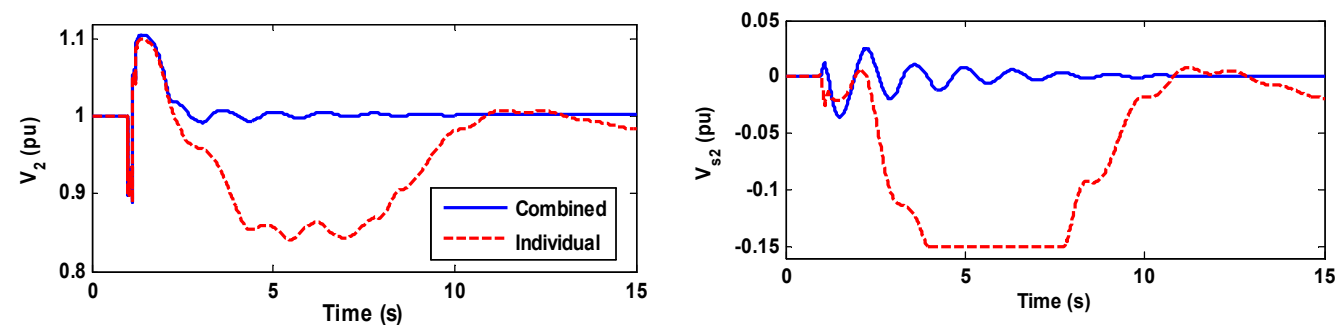

(a)
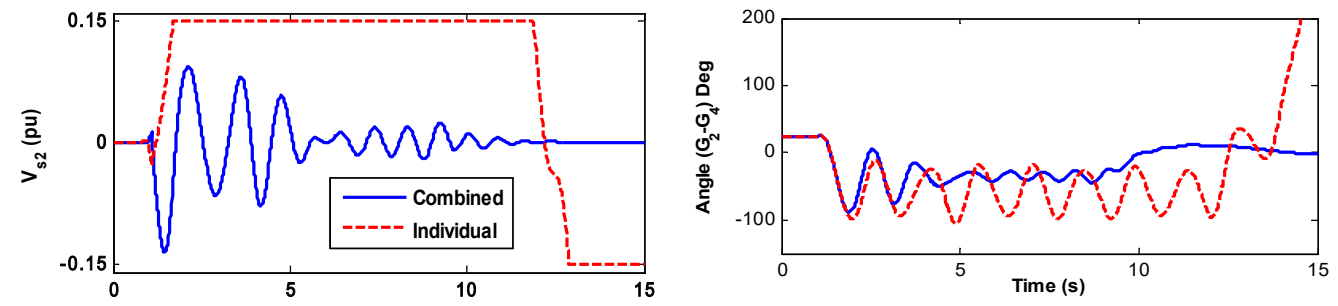

(b)

Fig. 9 Response of the PSS with a combined signal. (a) Major loss of load: 35\% at bus 9. (b) Major loss of generation: generator G1.

However, the combined signal can provide effective damping of the inter-area mode without adversely affecting the terminal voltage. As can be seen from the results, this leads to an improved transient response, while still ensuring the PSS is available for damping system oscillations. Fig.9(b) shows that even during this severe event, the PSS output is still far from its limits which indicates the effectiveness of the proposed solution.

\subsection{Impact on the common low frequency mode:}

It can be seen from Fig. 8(b) that the combined signal does not provide observability to the lowest natural frequency. This is because this low frequency mode is observable everywhere in the system and both amplitude and phase are almost the same for all outputs (i.e. all generators oscillate in the same direction with no phase shift). This observation has been shown, in several systems (e.g. Hydro-Québec and ERCOT) to be a general characteristic of the low frequency mode (below 01 Hz), which is generally called common low frequency mode $[45,46]$. Therefore, since both amplitude and phase are almost the same, a controller using the combined signal as input will fail to add positive damping to the common low frequency mode. These oscillations have an adverse effect on the frequency response during system upsets (increasing the frequency nadir). The frequencies and damping ratios of the common low frequency mode with both PSSs (with and without remote signal) versus the 
open loop case are given in Table 6. It appears that, without any PSS in the system, the mode has a significant amount of damping. This is due to the governor loops. However, adding more damping to this low frequency mode will significantly improve the systems overall stability, especially for perturbations involving excessive frequency excursions [45, 46]. This is confirmed by the simulation result of Fig. 10 where the load at Bus 9 has been reduced by $40 \%$. It is clear from this figure that better frequency response is obtained with individual signal. When the combined signal is used, the PSS at G2 has no effect on the common low frequency which causes a much deeper frequency nadir where the PSS at G4 saturates for two seconds. Hence, in systems having a common low frequency mode, one must be careful when using a combined signal as a PSS input.

\begin{tabular}{ccc}
\hline & Freq. & Damp. \\
\hline No PSS & 0.080 & 0.43 \\
\hline PSS with individual signal & 0.130 & 0.82 \\
\hline PSS with combined signal & 0.088 & 0.78 \\
\hline
\end{tabular}

Table 6 Common low frequency mode.
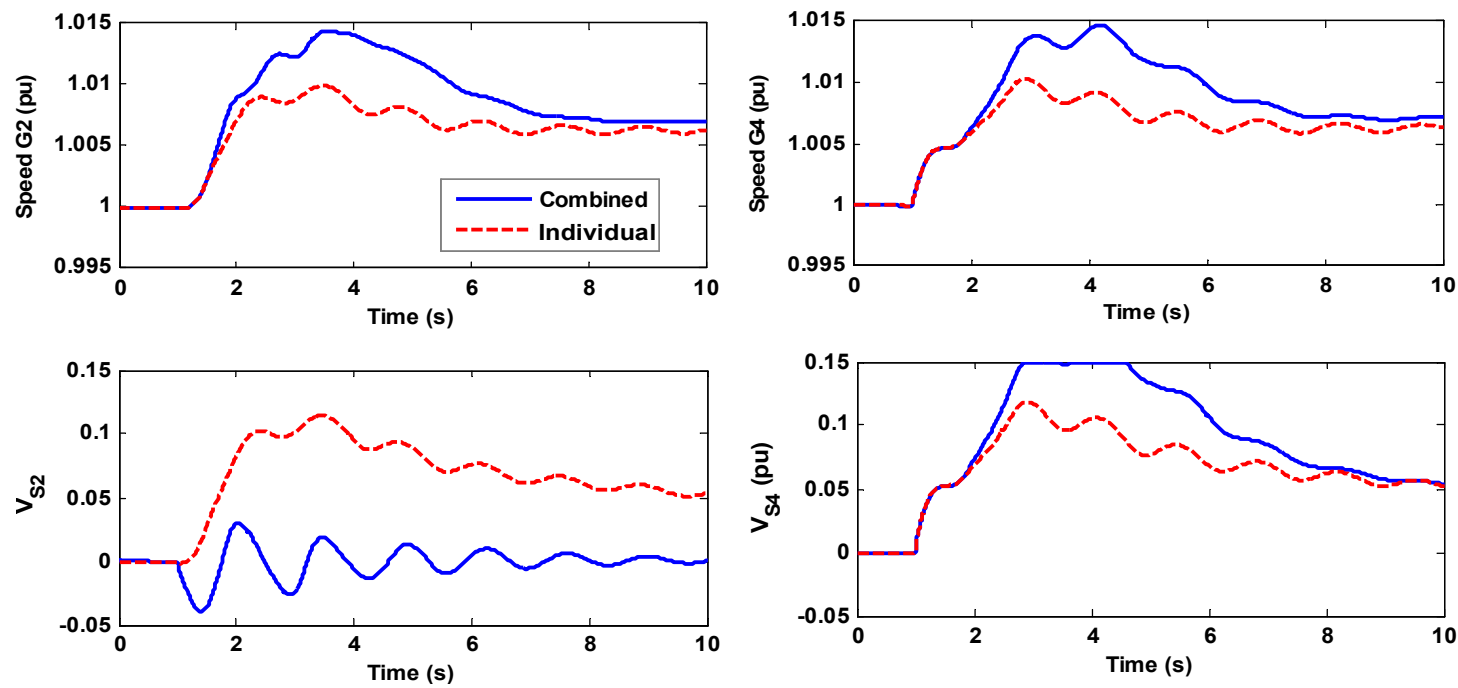

Fig. 10 Major loss of load: $40 \%$ at bus 9.

\subsection{Application to the New England power system}

In this part of the study, we have used the New England 10- machine 39-bus system shown in Fig. 11(a) to illustrate the effectiveness of our approach in a larger power system. Details of the system data are given in [47]. The system is implemented in MATLAB SPS. Each generator is equipped with a static exciter $\left(\mathrm{K}_{\mathrm{A}}=200, \mathrm{~T}_{\mathrm{A}}=0.015\right)$ and a steam turbine governor. The loads are modelled as constant impedances. This model of the New England system has been previously validated in [45]. The model is linearized around a nominal operating point. A small signal analysis showed that this system has several local and inter-area modes (nine modes) which are insufficiently damped; indeed, three eigenvalues have positive real parts showing that the system has unstable oscillations (see Fig. 11(b)). This is due to the high-gain fast-response AVR. To improve the damping of the oscillation modes, generators G4, G7 and G9 are equipped with PSSs. The block diagram of the PSS is shown in Fig. 12 [48]. There is an inter-area oscillation mode with $0.987 \mathrm{~Hz}$, in which generators G9 and G5 are the most involved. To show the performance of the proposed solution, a remote signal ( $\Delta \omega$ of G9, where the mode is highly observable) is used as input to the PSS at G4. Fig. 13(a) shows the responses of the combined signal and the individual signals without PSSs when $70 \%$ of generation at G6 is lost at time 1 s. Fig. 13(b) shows the results with PSSs when the load at Bus 29 is tripped. It is clear from these figures that the combined signal gives an excellent performance for both disturbances. By contrast, the PSS causes large excursions in generator voltage when the combined signal is not used in which the PSS output hits its limit as can be seen in Fig. 13(b). It should be noted that a negative gain is used at G4 when the individual signal $\left(\Delta \omega_{9}\right)$ is used as input. 

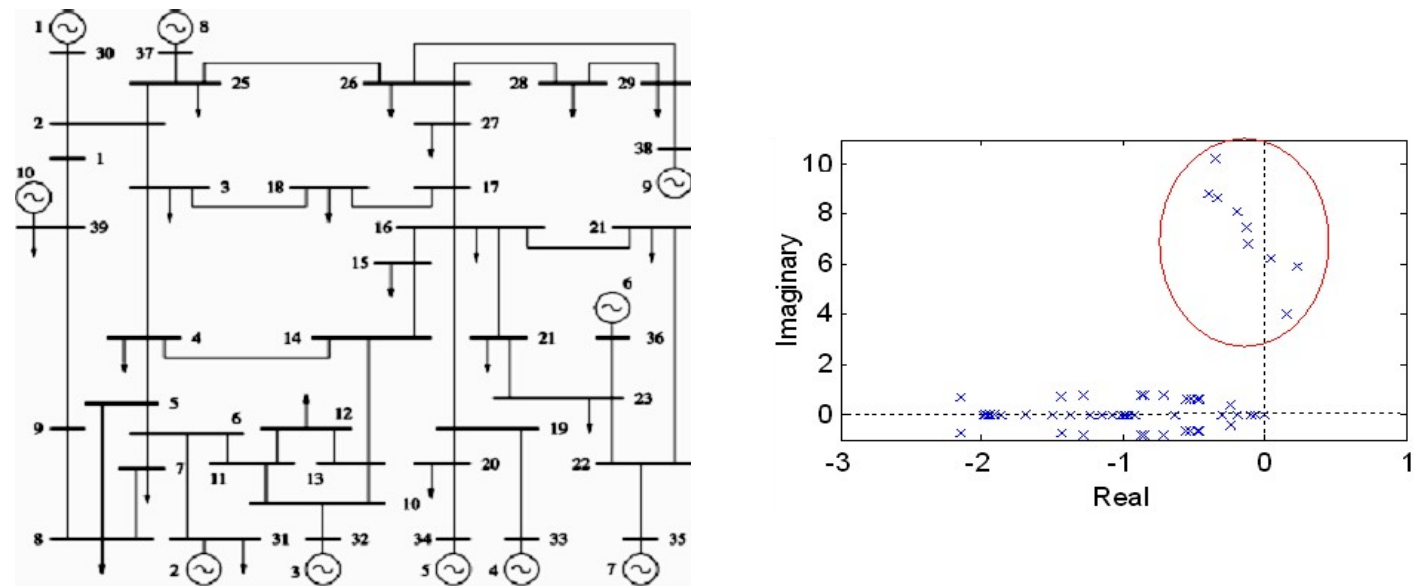

Fig. 11 New England 39-bus Test System and its eigenvalues.

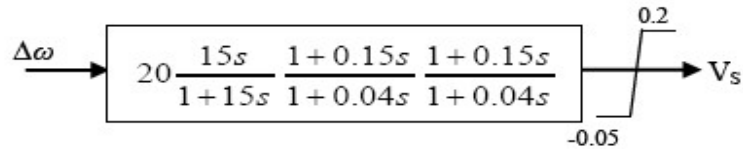

Fig. 12 PSS block diagram.

\subsection{Application to the Hydro-Québec system}

The excursion problem has been mitigated by the modern PSSs (e.g. PSS2B and PSS4B). However, since the primary task of these PSSs is to minimize the adverse torsional interactions and to provide adequate damping capabilities in the low frequency range [30,7], the advantage of these PSSs during severe system upsets is not sufficient as clearly shown in [7]. Based on the comparisons given in [7], the PSS4B can provide a good voltage recovery performance during major system upsets as compared to the PSS2B, which tends to saturate during severe events.

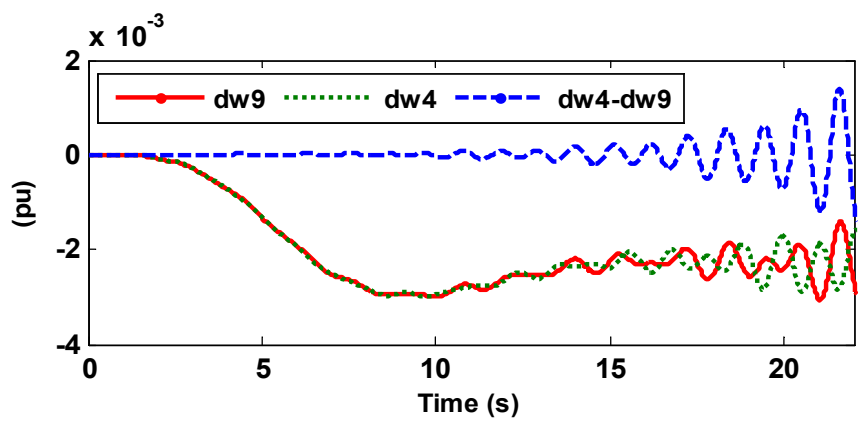

(a)
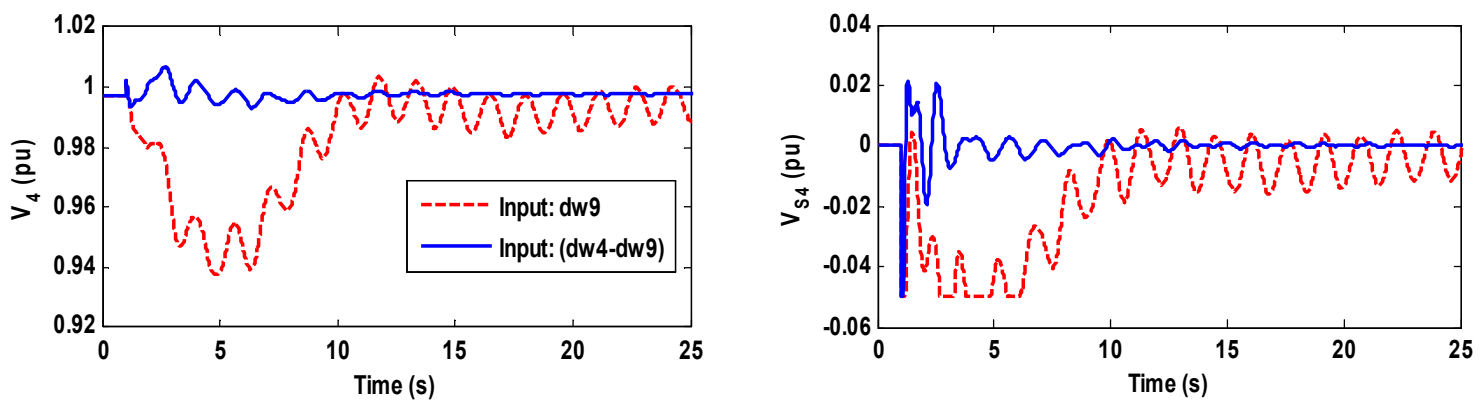

(b)

Fig. 13 Application to the New England system. (a) Responses of the combined signal and the individual signals. (b) Major loss of load: The load at bus 29 . 
The multi-band PSS included in the revised IEEE Std-421.5 as PSS4B has been developed by Hydro-Québec and ABB [49, 50] and installed in the Hydro-Québec system to provide adequate damping capabilities in the low frequency range. A simplified model of the Hydro-Québec system is proposed in this section to show the performance of the proposed solution and to make a comparison with the PSS4B. In this study, the system is represented by three areas implemented in MATLAB SPS. It contains four generators in the northwest (James Bay), two in the northeast (Churchill and Manic) and one in the south close to the load center (Montreal and Quebec). The model has been previously used in [51,52], where more details can be found. Each generator is equipped with a static exciter and a standard HYGOV. The system has several oscillation modes. There is an interarea mode between the northwest (for which LG2 is the largest plant) and northeast generating stations with nearly perfect phase opposition. A PSS4B was installed at LG2, and the performance of the candidate PSS1A (conventional $\Delta \omega$ PSS) and PSS4B is compared at Churchill plant following the trip of LG4 power plant. The speed shift between LG2 and Churchill is used to assess the proposed solution. Fig. 14(a) shows the responses of the combined signal and the individual signals without PSS when the LG4 is tripped (at $1 \mathrm{sec}$ ). The results clearly demonstrate the need for a PSS to stabilize the inter-area mode.

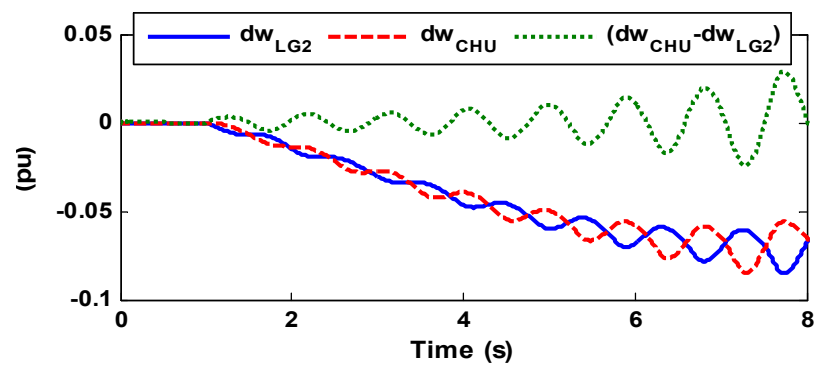

(a)
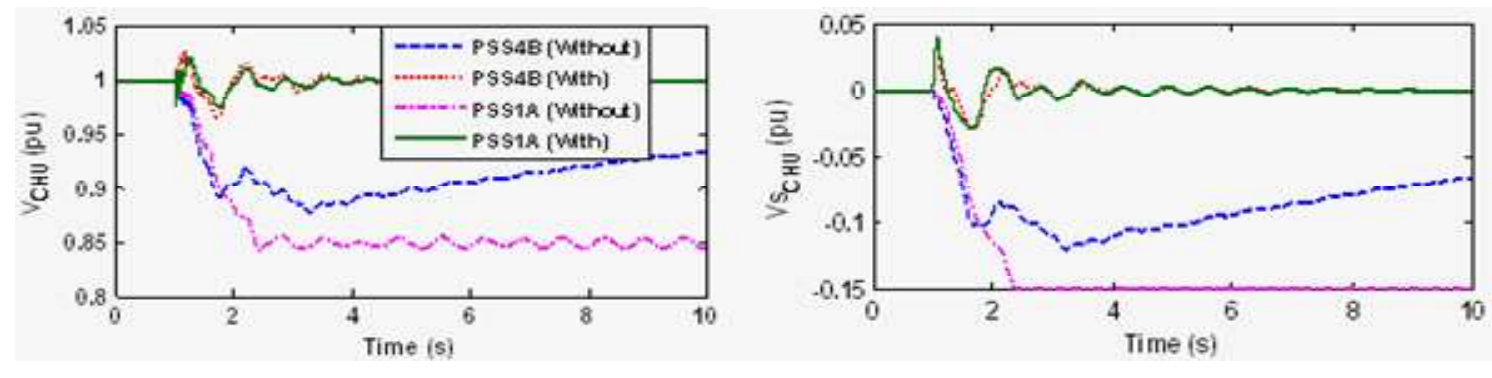

(b)

Fig. 14 Application to the Hydro-Québec system. (a) Responses of the combined signal and the individual signals. (b) Major loss of generation: LG4.

In Fig. 14(b), various PSSs with and without remote signal following the generation outage are assessed. Clearly, it appears that the combined signal can maintain a very good performance for both PSSs. Even though the action of PSS4B (without remote signal) causes significant voltage drop, subsequent voltage restoration is faster as compared to the conventional PSS, which it fails to recover from saturation. The parameters of the PSS1A are as mentioned previously in Fig. 2, while the parameters of the PSS4B are taken from [7].

\section{Discussion}

Because the offset and saturation problems are not taken into account when designing wide-area PSSs, in some works the design itself is done in such a way that it can increase the adverse effects on terminal voltage offset and the saturation of the controller is more likely to occur following large disturbances. For instance, in the design given in [14], a remote signal (speed variable) with a weighting factor is added to a local signal (speed variable) using a conventional double-lead-lag compensator structure for the controller (PSS1A). Since the two signals have the same sign, the excursion of the resulting signal during under- or over-frequency conditions will be larger than each of the individual signals in which the PSS saturation becomes more likely following a severe system upset. To assess this, time domain simulations of the test system were carried out in MATLAB SPS for various disturbances. The PSS output limits used in our simulation studies are 0.15 p.u. and -0.05 p.u., and each generator is equipped with a standard HYGOV. We found that the PSS saturates for various contingency scenarios (e.g., losing $45 \%$ of load at Bus 1 or at Bus 7, losing $70 \%$ of generation at Bus 4). The model has been validated by comparing its results with those available in the literature. 
One promising approach for applying wide-area PSSs is to use the wide-area control loop as a supplementary loop that works in parallel with the local loop (see Fig. 15(a)). This approach is well addressed in the literature [12, 13,15]. The first control signal at the first level (local loop) is to provide damping for local modes and the second control signal at the second level (wide-area loop) is to provide damping for the inter-area modes. The total control signal applied to the machine voltage reference is the sum of these two component control signals. Then, if the excursions of the two control signals $\left(V_{S R}\right.$ and $\left.V_{S L}\right)$ are in the same direction (i.e. have the same sign), the excursion of the total control signal $V_{S T}$ during transient conditions will be larger than each of the two control signals $\left(V_{S R}\right.$ and $\left.V_{S L}\right)$, where the PSS saturation is more likely to happen. In [53], a multi-loop design (more than two) is used. Usually, the local loop is a conventional PSS designed by classical methods (the wide-area loop is added to the existing PSS). Then, the total control signal can go into saturation during severe upsets due to the local loop even when the input of the wide-area controller is a combined signal. In order to investigate this problem, a wide-area loop with a combined input signal was added to the local PSS at G2 (Fig. 1). The controller of the wide-area loop is designed by the residue phase compensation method [13]. The required phase compensation is given by (6) and the block diagram of the obtained controller is given in Fig. 15(b).

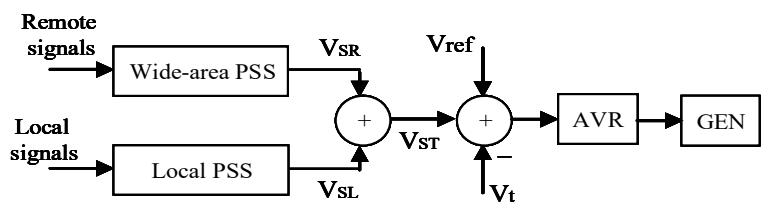

(a)

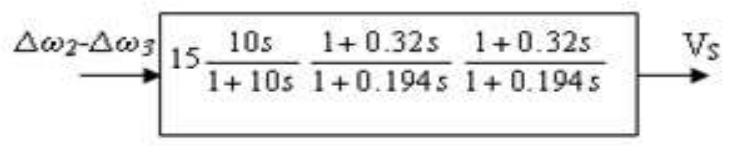

(b)

Fig. 15 Two-level PSS. (a) PSS structure. (b) Block diagram of the designed wide-area loop.

Fig. 16(a) shows the response of the three control signals ( $V_{S L}$ : local control signal, $V_{S R}$ : remote control signal and $V_{S T}$ : total control signal) when $60 \%$ of load at Bus 9 is lost. As can be seen, the total signal saturates for a while. To overcome this problem we propose to use lower output limits on the local PSS, as shown in Fig. 16(b), or to disconnect the local loop with a small washout time-constant when the total control signal blocks at one of its limits for a sufficient length of time.

As discussed previously, wide-area control is inherently more effective than local control for damping inter-area oscillations. However, this wide-area control does not improve the security of the transmission system because in the long run the additional capacity that it provides is used to increase the power transfer between areas rather than to increase security, see [54] for details. Then, the failure of a wide-area damping controller should be considered as a part of the N-1 criterion.

The ineffectiveness of a wide-area PSS following a large disturbance either because of a severe saturation or a programmed washout effect can be considered as a second failure that makes subsequent failures more likely leading to a possible blackout of the entire system, especially when the system is heavily stressed and the stability margins are small. Therefore, it is necessary to investigate the performance of the final design by extensive simulations for different contingencies; and not only for a short circuit on the transmission lines as in the most previous works, but also following severe system upsets which cause a significant imbalance between generation and load using a more detailed model with output limits and governors.

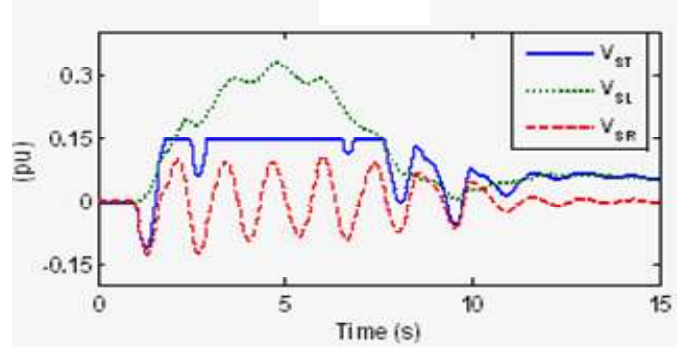

(a)

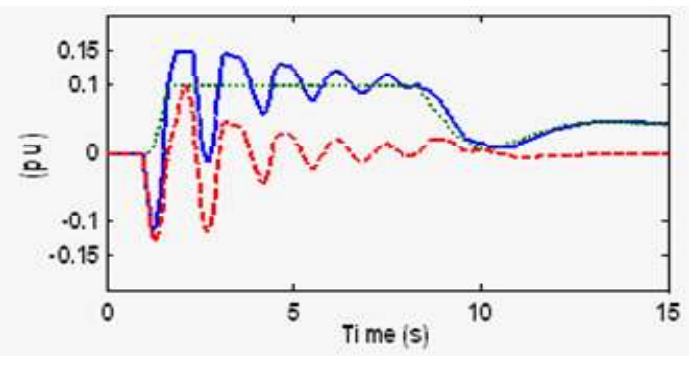

(b)

Fig. 16 Responses of the three control signals. (a) Without output limit on the local loop. (b) With lower output limit on the local loop ( \pm 0.1 ). 


\section{Conclusion}

Wide-area PSSs are an effective means of improving stability and thereby the reliability and robustness of today's interconnected power systems, but its implementation is costly. Therefore, the controller must be well configured and designed using proper control design techniques and procedures to ensure its cost-effectiveness. In this paper, the performance of widearea PSSs during major system upsets that cause large frequency and voltage excursions has been investigated and analysed via extensive simulations and detailed discussion. The results underline the importance of considering the excursion problem when designing wide-area PSSs. The main conclusions and findings drawn from this study are summarized as follows:

- The excursion problem is a real problem for wide-area PSSs due to the large magnitude of the observability index of the inter-area mode when a remote signal is used as input.

- The excursion problem is a limiting factor that needs to be considered in the overall design, especially because widearea PSSs are commonly designed using multiple signals (multi-input or multi-loop).

- The combined signal presented in this paper provides an excellent solution to the excursion problem. The main advantage of this signal is that it greatly reduces or even eliminates the excursions of the stabilizing signal and thus requires the use of a higher gain for improving damping. The second advantage is that this combined signal has the sensitivities to add damping for both the local mode (through the local signal) and the inter-area mode (through the remote signal). However, the combined signal has no sensitivity to add positive damping to the common low frequency mode.

It is hoped that this paper has demonstrated a real problem in the design stage of wide area PSSs, and will help to design effective controllers.

\section{References}

[1] IEEE/CIGRE Joint Task Force on Stability Terms and Definitions, "Definition and classification of power system stability," IEEE Trans. Power Syst., vol. 19, no. 3, pp. 1387-1401, Aug. 2004.

[2] Grigsby, L. L., Ed., The Electric Power Engineering Handbook, CRC Press/IEEE Press, 2001, ch. 11, Power System Dynamics and Stability.

[3] Kundur, P., Klein, M., Rogers, G., J., et al., "Application of power system stabilizers for enhancement of overall system stability", IEEE Trans. Power Syst., 1989, 4, (2), pp. 614-626.

[4] Kundur, P., "Power System Stability and Control", 1994, New York, Mc-Graw-Hill

[5] IEEE Standard 421.5, "IEEE Recommended Practice for Excitation System Models for Power System Stability Studies", 1992

[6] Dudgeon, G., W., Leithead, W., E., Dysko, A., et al., "The Effective Role of AVR and PSS in Power Systems, Frequency Response Analysis", IEEE Trans. Power Syst., 2007, 22, (4), pp. 1986-1994.

[7] Kamwa, I., Grondin, R., Trudel, G., "IEEE PSS2B versus PSS4B, The Limits of Performance of Modern Power System Stabilizers", IEEE Trans. Power Syst., 2005, 20, (2), pp. 903-915.

[8] Bollinger, K., E., Ao, S., Z., "PSS performance as affected by its output limiter", IEEE Trans. Energy Convers., 1996, 11, (1), pp. 118-124.

[9] Murdoch, A., Venkataraman, S., Sanchez-Gasca, J. J., et al., "Practical application considerations for power system stabilizer (PSS) controls," in Proc. IEEE PES Summer Meeting, vol. 1, Jul. 1999, pp. 83-87.

[10] Yang, X., Feliachi, A., "Stabilization of inter-area oscillation modes through excitation systems", IEEE Trans. Power Syst., 1994, 9, (1), pp. 494-502.

[11] Taylor, C. W., Erickson, D. C., Martin, K. E., Wilson, R. E., and Venkatasubramanian, V., " WACS-wide-area stability and voltage control system, R\&D and online demonstration", Proc. of the IEEE, vol. 93, no. 5, pp. 892-906, May 2005.

[12] Kamwa, I., Grondin, R., Hebert, Y., "Wide-area measurement based stabilizing control of large power systems-a decentralized/hierarchical approach", IEEE Trans. Power Syst., 2001, 16, (1), pp. 136-153

[13] Aboul-Ela, M., E., Sallam, A., A., McCalley, J., D., et al., "Damping controller design for power system oscillations using global signals", IEEE Trans. Power Syst., 1996, 11, pp. 767-773

[14] Chow, J., H., Sanchez-Gasca, J. J., Ren, H., et al., "Power system damping controller design using multiple input signals", IEEE Contr. Syst. Mag., 2000, 20, (4), pp. 82-90.

[15]Zhang, Y., Bose, A., "Design of wide-area damping controllers for interarea oscillations", IEEE Trans. Power Syst., 2008, 23, (3), pp. 1136-1143

[16] Yao, W., Jiang, L., Wen, J., et al.: 'Wide-area damping controller for power system interarea oscillations: a networked predictive control approach', IEEE Trans. Control Syst. Technol., 2015, 23, (1), pp. 27-36

[17] R. Yousefian and S. Kamalasadan, "Hybrid transient energy function based real-time optimal wide-area damping controller," IEEE Trans. On Industry Applications, vol. PP, pp. 1-1, 2016.

[18] T. Surinkaew and I. Ngamroo, "Hierarchical co-ordinated wide area and local controls of DFIG wind turbine and PSS for robust power oscillation damping," IEEE Trans. Sustain. Energy, vol. 7, no. 3, pp. 943-955, Jul. 2016.

[19] V. V. G. Krishnan, S. C. Srivastava, and S. Chakrabarti, "A robust decentralized wide area damping controller for wind generators and FACTS controllers considering load model uncertainties," IEEE Trans. Smart Grid, vol. 9, no. 1, pp. 360-372, Apr. 2018. 
[20] T. Surinkaew and I. Ngamroo, "Adaptive signal selection of wide-area damping controllers under various operating conditions," IEEE Trans. Ind. Inform., vol. 14, no. 2, pp. 639-651, Feb. 2018.

[21]B. Chaudhuri, R. Majumder, and B. C. Pal, "Wide-area measurement based stabilizing control of power system considering signal transmission delay,” IEEE Trans. Power Syst., vol. 19, no. 4, pp. 1971-1979, Nov. 2004.

[22] L. Cheng, G. Chen, W. Gao, F. Zhang, and G. Li, "Adaptive time delay compensator (ATDC) design for wide-area power system stabilizer,” IEEE Trans. Smart Grid, vol. 5, no. 6, pp. 2957-2966, Nov. 2014.

[23] M. Beiraghi and A. M. Ranjbar, "Adaptive delay compensator for the robust wide-area damping controller design," IEEE Trans. Power Syst., vol. 31, no. 6, pp. 4966-4976, Nov. 2016.

[24]B. P. Padhy, S. C. Srivastava, and N. K. Verma, "A wide-area damping controller considering network input and output delays and packet drop,” IEEE Trans. Power Syst., vol. 32, no. 1, pp. 166-176, Jan. 2017.

[25] Y. Li, Y. Zhou, F. Liu, Y. Cao, and C. Rehtanz, "Design and implementation of delay-dependent wide-area damping control for stability enhancement of power systems," IEEE Trans. Smart Grid, vol. 8, no. 4, pp. 1831-1842, Jul. 2017.

[26] M. C. Obaiah, B. Subudhi, "Power System Supplementary Damping Controllers in the Presence of Saturation," 2017 IEEE Power and Energy Conference at Illinois (PECI), Champaign, IL, USA, 2017.

[27] J. Fang, W. Yao, Z. Chen, J. Wen, C. Su, S. Cheng, "Improvement of wide-area damping controller subject to actuator saturation: a dynamic antiwindup approach," IET Gener. Transm. Distrib., vol. 12, no. 9, pp. 2115-2123, 2018.

[28] T. Lin, G. Ding, R. Chen, B. Chen, X. Xu, "Wide-area damping state feedback and output feedback controller design strategy considering time delays and actuator saturation based on parametric Lyapunov theory," AIP Advances, vol. 9, no. $3,2019$.

[29] M. C. Obaiah, B. Subudhi, “Anti-windup compensator design for power system subjected to time-delay and actuator saturation," IET Smart Grid, vol. 2, no. 1, pp. 106-114, 2019.

[30] Kundur, P., Berube, G., R., Hajagos, L., M., et al., "Practical Utility Experience with and Effective Use of Power System Stabilizers", IEEE PES Meeting, Vol. 3, Jul. 2003, pp. 1777- 1785.

[31] IEEE Standard 421.5., "IEEE Recommended Practice for Excitation System Models for Power System Stability Studies", 2006 (Revision of IEEE Standard 421.5-1992).

[32] Rogers, G., "Power System Oscillations", (Boston, MA, Kluwer, 2000)

[33] B. Pal, B. Chaudhuri, Robust Control in Power Systems. New York, Springer Inc., 2005.

[34] Pagola, F., L., Perez-Arriaga, I., J., Verghese, G., C., "On sensitivities, residues and participations, applications to oscillatory stability analysis and control", IEEE Trans. Power Syst., 1989, 4, (1), pp. 278- 285

[35] Law, K., T., Hill, D., J., Godfrey, N., R., "Robust controller structure for coordinated power system voltage regulator and stabilizer design", IEEE Trans. Control Syst. Technol., 1994, 2, (3), pp. 220-232

[36] Klein, M., Rogers, G., J., Moorty, S., et al., "Analytical investigation of factors influencing power system stabilizer performance", IEEE Trans. Energy Convers., 1992, 7, (3), pp. 382-390

[37] Bollinger, K. E., Gu, W., Norum, E., "Accelerating power versus electrical power as input signals to power system stabilizers", IEEE Trans. Energy Convers., 1991, 6, (4), pp. 620-626

[38] Watson, W., Manchur, G., "Experience with Supplementary Damping Signals for Generator Static Excitation Systems", IEEE Trans., 1973, PAS-92, pp. 199-203

[39] deMello, F., P., Hannett, L., N., Undrill, J., M., "Practical Approaches to Supplementary Stabilizing from Accelerating Power", IEEE Trans., 1978, PAS-97, pp. 1515-1522

[40] Andersson, G., " Dynamics and Control of Electric Power Systems", Lecture 227-0528-00, ITET ETH, 2012

[41]ENTSO-E., "Future system inertia", ENTSO Report, 2013.

[42]ENTSO-E., "Dispersed generation impact on CE region security", ENTSO-E SPD Report, 2014.

[43] Yang, X., Feliachi, A., Adapa, R., "Damping enhancement in the western U.S. power system, a case study", IEEE Trans. Power Syst., 1995, 10, (3), pp. 1271-1278

[44] Stahlhut, J., W., Browne, T., J., Heydt, G., T., et al., "Latency viewed as a stochastic process and its impact on wide area power system control signals", IEEE Trans. Power Syst., 2008, 23, (1), pp. 84-91

[45] Moeini, A., Kamwa, I., "Analytical Concepts for Reactive Power Based Primary Frequency Control in Power Systems", IEEE Trans. Power Syst., 2015, 99, pp. 1-14

[46] Grondin, R., Kamwa, I., Soulieres, L., et al., "An approach to PSS design for transient stability improvement through supplementary damping of the common low-frequency", IEEE Trans. Power Syst., 1993, 8, (3), pp. 954-963.

[47] Pai, M., A., "Energy Function Analysis for Power System Stability". (Norwell, MA, Kluwer, 1989)

[48] IEEE PES Task Force on Benchmark Systems for Stability Controls "Report on the 68-bus system (New England / New York Test System)", Ver 4.0, Jun, 2014.

[49] Grondin, R., Kamwa, I., Trudel, G., Taborda, J., Lenstroem, R., Gerin-Lajoie, L., Gingras, J. P., Racine, M., Baumberger, H., "The multi-band PSS, A flexible technology designed to meet opening markets", in Proc. CIGRÉ 2000, Paris, France. Paper 39-201.

[50] Grondin, R., Kamwa, I., Trudel, G., Gérin-Lajoie, L., Taborda, J., "Modeling and closed-loop validation of a new PSS concept, the multi-band PSS", presented at the 2003 IEEE/PES General Meeting, Panel Session on New PSS Technologies, Toronto, ON, Canada.

[51] Kamwa, I., Samantaray, S., Joos, G., "Optimal integration of disparate C37.118 PMUs in wide-area PSS with electromagnetic transients", IEEE Trans. Power Syst., 2013, 28, (4), pp. 4760-4770 
[52] Ghahremani, E., Kamwa, I., "Local and Wide-Area PMU-Based Decentralized Dynamic State Estimation in MultiMachine Power Systems", IEEE Trans. Power Syst., 2016, 31, (1), pp. 547-562

[53] Kamwa, I., Gerin-Lajoie, L., Trudel, G., "Multi-loop power system stabilizers using wide-area synchronous phasor measurements", in Proc. American Control Conf., June 1998, 5, pp. 2963-2967.

[54] Kirschen, D., Strbac, G., "Why Investments Do Not Prevent Blackouts", The Electricity Journal, 2004, pp. 29-36 